\title{
Principi di immunobiologia del trapianto ed attivazione della risposta immune
}

Giornale di Tecniche Nefrologiche e Dialitiche 2019, Vol. 3I (I) 65-70

(C) The Author(s) 2019

Article reuse guidelines:

sagepub.com/journals-permissions DOI: $10.1177 / 0394936218823554$

journals.sagepub.com/home/gtn

\author{
Aris Tsalouchos' e Maurizio Salvadori²
}

\begin{abstract}
To achieve an effective immune response it is important that $T$ cells can recognize a wide variety of non-self antigens; this allows for restrained immune activation and subsequent antigen-specific killing. This task is accomplished through the generation of a repertoire of $T$ cells in a single individual with specificity for an enormous number of potential foreign antigens presented as peptides on the surface of major histocompatibility complex (MHC) molecules. Variations in MHC structure among individuals increase the variety of peptides that can be presented to $T$ cells; this mechanism protects the species as a whole by ensuring adequate $\mathrm{T}$-cell responses to a given foreign organism. Although slightly different, these MHC polymorphisms expressed in the donor kidney are recognized after kidney transplantation between non genetically-identical humans, and induce alloresponses that in the absence of immunosuppression result in rejection of the allograft. In this chapter, we review basic immunological principles important to the field of kidney transplantation.
\end{abstract}

\section{Keywords}

Kidney transplantation, Major histocompatibility complex, Antigen recognition, T-cell activation, B cell activation

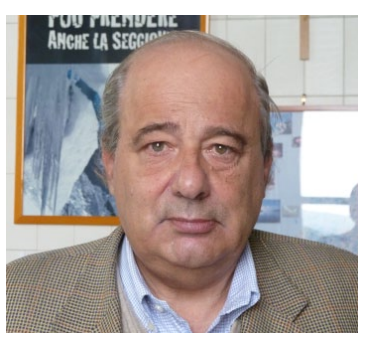

\section{Introduzione}

Il trapianto di rene rappresenta l'alternativa indiscutibilmente migliorativa per i pazienti con insufficienza renale terminale. Tuttavia, gli ostacoli principali che tale terapia presenta sono la scarsa disponibilità di organi, a fronte di una richiesta sempre crescente, e il problema del rigetto sia acuto che cronico. Il trapianto, eccetto i pochissimi casi di trapianto fra gemelli monozigoti, è materiale non-self e l'organismo tende a rifiutarlo come qualsiasi altro patogeno. La comprensione del perché si verifica tale meccanismo è essenziale anche per comprendere ed attuare le terapie immunosoppressive atte a controllare il rigetto.

Per questo motivo, in questa rassegna verranno prima trattate le basi immunologiche per le quali si possono manifestare fenomeni di rigetto, e in un secondo momento i meccanismi per i quali si ha attivazione della risposta immunitaria, prendendo in considerazione le sue diverse componenti.

In generale, occorre distinguere 4 tipi di trapianto: a) L'autotrapianto, che consiste nel trapiantare un organo o un tessuto da una parte all'altra dello stesso soggetto. Non si verificano fenomeni immunologici.

b) L'isotrapianto, che consiste nel trapiantare un organo o tessuto fra soggetti della stessa specie, come è il caso dei gemelli monocoriali. Raramente si verifica attivazione immunologica.

c) L'allotrapianto o omotrapianto, che consiste nel trapiantare organi o tessuti fra membri non geneticamente uguali della stessa specie. Esiste attivazione immunologica ed è il trapianto che più ci riguarda in questo contesto.

d) Lo xenotrapianto, che avviene fra soggetti di specie diversa. L'attivazione immunologica è particolarmente intensa ed aggressiva.

'Azienda UsI Toscana Centro, S.O.S. Nefrologia e Dialisi, Ospedale SS Cosma e Damiano, Pescia, Italia

2Professore di Nefrologia già Direttore Nefrologia e Trapianto, Azienda Ospedaliera Careggi, Firenze, Italia

\section{Corrispondenza:}

Professor Maurizio Salvadori, già Direttore Nefrologia e Trapianto,

Azienda Ospedaliera Careggi, Firenze, Italia.

E-mail: maurizio.salvadoril@gmail.com 


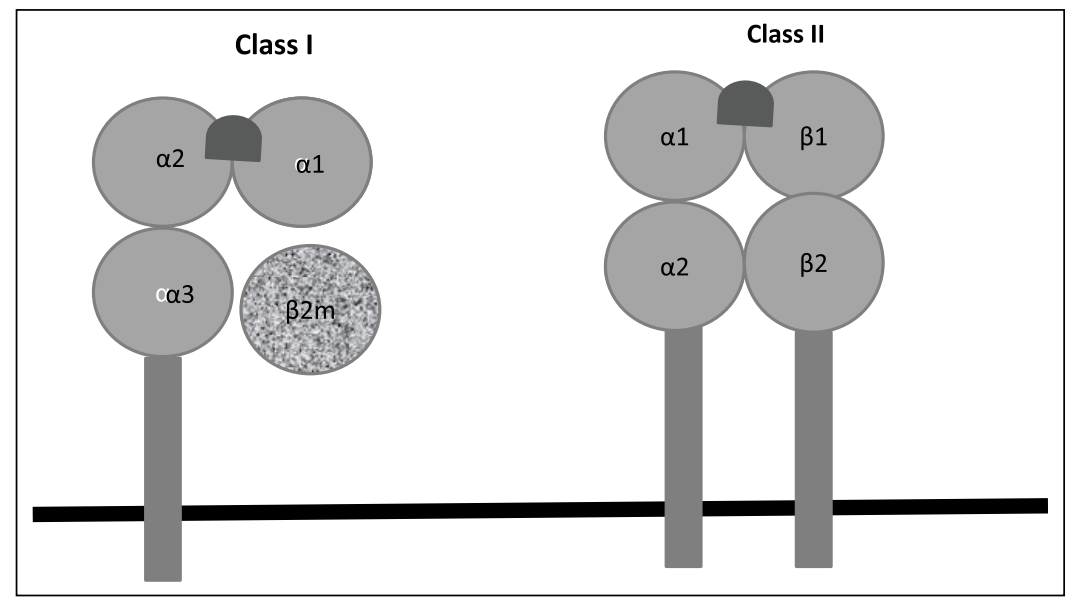

Figura I. Molecole HLA di classe I e II sulla membrana cellulare

\section{Immunobiologia del trapianto}

In condizioni normali l'organismo è tollerante nei confronti dei propri tessuti, cosa che non si verifica nell'allotrapianto perché l'organo trapiantato esprime antigeni che non sono riconosciuti dalle cellule immunitarie di chi riceve il trapianto. Sono stati identificati diversi antigeni che svolgono un ruolo importante nel trapianto, fra cui le molecole del sistema maggiore di istocompatibilità (MHC), gli antigeni del gruppo sanguigno $\mathrm{AB}$, antigeni propri dell'endotelio e dei monociti, ed altri antigeni minori di istocompatibilità.

Gli antigeni che rivestono maggiore importanza sono quelli del sistema MHC, che nell'uomo codificano gli antigeni del sistema HLA.

Nella specie umana la regione HLA è localizzata nel braccio corto del cromosoma 6 e codifica due gruppi di molecole che sono poi presenti sulle differenti cellule: le molecole di classe I e di classe II (figura 1). ${ }^{1}$

Le molecole di classe I consistono in due catene polipeptidiche simili a quelle delle immunoglobuline. La catena pesante contiene la parte antigenica. La catena leggera è la $\beta 2$-microglobulina. La sequenza variabile amminoacidica che conferisce l'immunogenicità è contenuta nel primo e secondo dominio. Le molecole di classe I sono espresse su quasi tutte le cellule nucleate. Particolarmente ricchi sono i linfociti. Si distinguono tre loci per la classe I, HLA-A, HLA-B e HLA-C. Attualmente, mediante lo studio del DNA, sono stati definiti più di 8,000 alleli per la classe I, anche se molti di questi non sono clinicamente rilevanti nel trapianto d'organo.

Le molecole della classe II del sistema HLA sono formate da due catene polipeptidiche glicosilate chiamate $\alpha$ e $\beta$. La regione cromosomica che contiene i geni di classe II è chiamata HLA-D e sono stati identificati più di 2,000 alleli mediante lo studio del DNA. Gli antigeni delle molecole di classe II sono espressi solo sui linfociti B, sui monociti e macrofagi, cellule dendritiche e linfociti $\mathrm{T}$ attivati. Stimoli flogistici possono indurre l'espressione di molecole di classe II su endotelio ed altre cellule. I peptidi immunogenici sono presentati dai domini $\alpha 1$ e $\beta 1$ delle molecole di classe II.

La tipizzazione HLA sia di un ricevente che di un donatore è essenziale per garantire che il trapianto avvenga col maggior grado possibile di identità fra HLA del donatore e del ricevente, e per limitare al massimo le reazioni immunologiche che porterebbero a rigetto dell'organo. La tipizzazione si effettua col match di linfociti del soggetto con tutti gli anticorpi possibili diretti contro il sistema HLA. Tali anticorpi provengono dagli screening effettuati su migliaia di campioni di siero di multipare.

Quando, come è il caso di trapianti fra viventi, si desidera un elevato grado di specificità, si ricorre alla cultura mista linfocitaria. I linfociti di due soggetti sono posti in coltura e la proliferazione linfocitaria si verifica in 5-7 giorni ed è misurata dall'incorporazione di timidina triziata. Una delle due popolazioni (quella del donatore) viene precedentemente irradiata per impedirne la proliferazione.

\section{Antigeni non-HLA}

Il verificarsi di rigetti iperacuti umorali in soggetti con compatibilità $\mathrm{AB} 0$ e con cross-match negativo per gli anticorpi HLA ha fatto pensare al ruolo di antigeni nonHLA. Questi target antigenici sono espressi sulle cellule dell'organo trapiantato, in particolare sull'endotelio ed epitelio, e sono conosciuti come MICA o MICB. ${ }^{2}$ Altri antigeni sono stati identificati come la vimentina, la miosina, il collageno V, e il recettore di tipo I dell'angiotensina II.

\section{Antigeni $A B O$}

Gli antigeni dei gruppi sanguigni sono prontamente riconosciuti da anticorpi naturali chiamati emoagglutinine perché provocano l'agglutinazione dei globuli rossi. 
Tabella I. Generazione della risposta immune nel trapianto.

I. Danno da ischemia-riperfusione

2. Presentazione dell'antigene

3. Attivazione della cellula $\mathbf{T}$

4. Funzioni effettrici

5. Danno del trapianto

Sono rilevanti nel trapianto perché sono anche espressi su altri tipi di cellule fra cui le cellule endoteliali. Provocano rigetti iperacuti di tipo vascolare, qualora la loro identità o compatibilità non sia rispettata.

Recentemente, per procedere al trapianto anche in caso di incompatibilità $\mathrm{AB} 0$, in particolare per donatori viventi, diversi tipi di desensibilizzazione si sono dimostrati efficaci. Oltre la plasmaferesi, alte dosi endovena di immunoglobuline e anticorpi monoclonali anti-CD20 hanno fornito eccellenti risultati, anche a lungo termine, dopo il trapianto renale.

\section{Attivazione della risposta immune post-trapianto}

Dopo un trapianto di organo, ed in particolare di rene, eccetto i pochi casi di isotrapianti, le cellule $\mathrm{T}$ del ricevente riconoscono gli antigeni non-self (ovvero i peptidi estranei) presentati sulla superficie delle molecole del MHC localizzate sull'organo trapiantato (tabella 1$){ }^{3}$

\section{Danno da ischemia-riperfusione}

Il danno da ischemia-riperfusione, connesso alle manovre di prelievo e di trapianto del rene ed all'inevitabile periodo di ischemia fredda, è il primo danno che viene inferto all'organo trapiantato e la sua patogenesi coinvolge fattori biochimici, cellulari, connessi all'endotelio, per i quali si realizza una condizione di flogosi. ${ }^{4}$

$\mathrm{Si}$ ha la liberazione di citochine, fra cui il TNF, le interleuchine (IL) 1 e 6, e l'interferone (IFN). Questi eventi rendono l'organo trapiantato una sede di danno e di flogosi che, a sua volta, stimola la migrazione di cellule atte a presentare l'antigene (antigen presenting cells, APC) del donatore dal trapianto al tessuto linfoide del ricevente, con conseguente reclutamento dei leucociti. Viene così stimolata l'immunità adattiva con il coinvolgimento delle cellule T e le cellule B produttrici di anticorpi.

\section{Presentazione dell'antigene}

- APC

Le APC sono cellule capaci di attivare i linfociti $\mathrm{T}$. Cellule dendritiche, macrofagi e cellule B sono considerate "APC professionali". Nel trapianto o nei tessuti circostanti, le cellule dendritiche o del donatore o

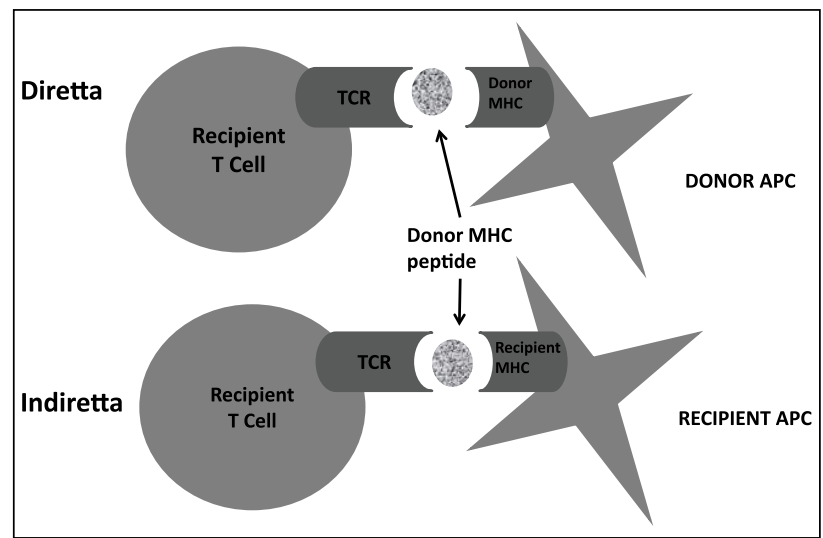

Figura 2. Presentazione diretta ed indiretta dell'antigene

del ricevente, si attivano e muovono verso le aree delle cellule $\mathrm{T}$ degli organi linfoidi secondari. Il movimento delle cellule $\mathrm{T}$ dendritiche e delle cellule $\mathrm{T}$ native è coordinato in modo da portarle entrambe nelle aree $\mathrm{T}$ degli organi linfoidi secondari dove l'attivazione delle cellule $\mathrm{T}$ è estremamente efficace. Una volta attivate, le cellule $\mathrm{T}$ lasciano gli organi linfoidi secondari, entrano nel sangue e si recano nei tessuti oggetto di flogosi, ovvero il trapianto nel caso in questione.

- Modalità di riconoscimento dell'antigene

Le cellule $\mathrm{T}$ del ricevente riconoscono l'antigene esogeno per via diretta o indiretta (figura 2). ${ }^{5} \mathrm{La}$ presentazione diretta dell'antigene coinvolge il riconoscimento da parte delle cellule $\mathrm{T}$ del ricevente dei peptidi del $\mathrm{MHC}$ del donatore ubicati sulla superficie delle APC del donatore.

La presentazione indiretta dell'antigene è il meccanismo fisiologico di riconoscimento di un antigene estraneo. L'antigene estraneo è captato dalle APC del ricevente, viene processato internamente, e quindi presentato come peptide sulle molecole del MHC.

\section{Attivazione delle cellule $T$}

La risposta immune inizia con il riconoscimento delle molecole non-self del sistema MHC da parte delle cellule $\mathrm{T}$ che vengono in tal modo attivate.

- Recettore delle cellule T

Le cellule $\mathrm{T}$ hanno in superficie un recettore che consiste in due differenti catene polipeptidiche legate da un ponte disulfurico. Questo recettore delle cellule T (TCR) non riconosce gli antigeni nelle loro condizioni native, ma li riconosce quando questi sono legati ad una molecola del sistema MHC.

Il processo di attivazione avviene attraverso due fasi, o segnali. 


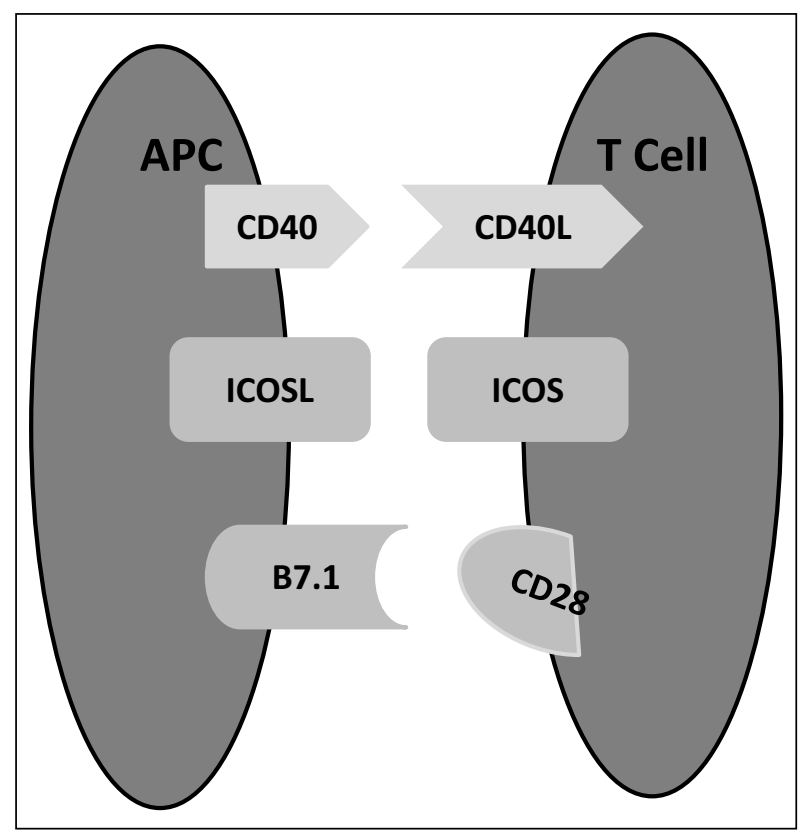

Figura 3. Segnali di co-stimolazione fra cellula presentante l'antigene e linfocita $T$

- Legame del TCR con l'antigene: segnale 1

Il legame fra TCR e peptide dell'MHC è la prima tappa, ma non è sufficiente perché occorre anche l'attivazione di un'altra struttura sulla superficie della cellula $\mathrm{T}$ chiamata complesso CD3.

- Co-stimolazione della cellula T: segnale 2

Anche il legame del complesso TCR-CD3 al complesso peptide-MHC sulle APC necessita di ulteriori segnali di co-stimolazione.

Molte molecole sono presenti sulla superficie della cellula T o della APC con i loro ligandi. Dall'interazione completa di queste strutture (figura 3 ), deriva l'espansione clonale delle cellule T. Il CD28 è presente sulle cellule T ed il suo ligand B.1 e B7.2 sulle APC. Altro legame è quello fra la molecola ICOS (cellula T) e l'ICOS ligand sulle APC. Altro legame è quello fra la struttura CD40 (sulle APC) ed il CD40 ligand (cellula T). La presenza ed il legame di tutte queste strutture rappresenta il secondo segnale e permette l'attivazione delle cellule $\mathrm{T}^{6}$

- Espansione clonale e differenziazione delle cellule T

Una risposta immune efficace e completa richiede l'espansione clonale e la differenziazione delle cellule $\mathrm{T}$. Questi processi sono mediati da specifiche molecole chiamate citochine. Una delle più importanti è l'IL-2 che si lega al suo recettore (IL-2R) anch'esso ubicato sulle cellule T. Questo fatto innesca tutta una serie di reazioni per le quali si ha una proliferazione adeguata. La tabella 2 fornisce un elenco completo delle citochine coinvolte.

\section{- Cellule T della memoria}

Nel trapianto, la precedente sensibilizzazione all'MHC del donatore da parte delle cellule $\mathrm{T}$ del ricevente è associata a formazione di cellule $\mathrm{T}$ della memoria che facilitano il fenomeno del rigetto con successivo fallimento del trapianto.

Le cellule della memoria possono essere state generate in seguito a precedenti trasfusioni, gravidanze o a un trapianto. Le cellule della memoria sono più facilmente attivate delle altre cellule $T$, entrano facilmente nei tessuti affetti da flogosi e secernono una gran quantità di citochine. ${ }^{7}$

\section{Funzioni effettrici}

Le cellule $T$, una volta attivate ed espanse, esercitano le loro funzioni effettrici che possono risultare nella distruzione del trapianto. Le cellule CD4 Thelper rilasciano numerose citochine che danneggiano l'organo. Le citochine delle cellule $\mathrm{T}$ possono agire direttamente sulle cellule parenchimali o indirettamente attraverso i loro effetti sull'endotelio e sulla circolazione. TNF- $\alpha$ e TNF- $\beta$ hanno un effetto citotossico sui recettori ubicati sulle cellule endoteliali o tubulari. L'IFN- $\gamma$ promuove l'espressione delle molecole di classe I e II sulle cellule del trapianto.

Sempre le cellule CD4 possono esercitare direttamente un'azione citolitica simile a quella esercitata dalle cellule CD8. Infine, le cellule CD4 possono stimolare le cellule B ad incrementare la produzione di anticorpi.

\section{- Differenziazione delle cellule T citolitiche}

Quasi tutte le cellule $\mathrm{T}$ con attività citolitica appartengono alla classe CD8. L'attività citolitica è esercitata da due meccanismi che richiedono sempre un contatto cellula-cellula. Possono rilasciare perforina e granzyme B. La perforina, attivato il complemento, induce la realizzazione di pori sulla membrana. $\mathrm{Si}$ ha sinergismo fra le due molecole. La perforina permette al granzyme B di entrare nella cellula; questo, una volta entrato, induce la morte programmata della cellula. Viene anche prodotta una terza proteina citotossica, la granulisina, che ugualmente induce apoptosi cellulare. ${ }^{8}$

L'altro meccanismo citolitico avviene attraverso l'interazione di Fas e Fas ligand (FasL). Questa interazione fra $\mathrm{T}$ citolitici e cellule bersaglio induce l'apoptosi di queste ultime. Le apoptosi descritte a carico delle cellule bersaglio avvengono attraverso l'attivazione della cascata delle caspasi.

\section{- Attivazione dei macrofagi}

In risposta a citochine come IL-1 e IL-6 i macrofagi sono reclutati nel trapianto. 
Tabella 2. Citochine coinvolte nella risposta immune.

\begin{tabular}{|c|c|c|}
\hline Citochine & Cellule generatrici & Attività biologica \\
\hline IL-I & Macrofagi, cellule dendritiche, cellule NK & $\begin{array}{l}\text { Pro-infiammatoria, facilita l'espressione delle } \\
\text { molecole di adesione }\end{array}$ \\
\hline IL-2 & Cellule $\mathbf{T}$ attivate & $\begin{array}{l}\text { Proliferazione cellule } \mathbf{T} \text {, produzione anticorpi da } \\
\text { cellule } \mathrm{B} \text {, attività citolitica }\end{array}$ \\
\hline IL-4 & Cellule $\mathbf{T}$ attivate & $\begin{array}{l}\text { Proliferazione cellule T e B attivate, up-regolazione } \\
\text { del MHC sulle cellule B }\end{array}$ \\
\hline IL-6 & Cellule T, macrofagi, endotelio & Pro-infiammatoria \\
\hline IL-I 0 & Cellule T, macrofagi & Antiinfiammatoria, inibizione delle cellule NK \\
\hline IL-I 2 & Macrofagi, cellule dendritiche & $\begin{array}{l}\text { Pro-infiammatoria, differenziazione cellule T, attività } \\
\text { cellule NK e citolitiche, produzione IFN- } \gamma \text { e TNF- } \alpha\end{array}$ \\
\hline IL-I 5 & Cellule epiteliali, macrofagi & $\begin{array}{l}\text { Proliferazione cellule NK, proliferazione cellule T, } \\
\text { generazione cellule T della memoria }\end{array}$ \\
\hline IL-I 7 & Cellule T & Pro-infiammatoria, produzione di citochine \\
\hline IFN- $\boldsymbol{\gamma}$ & $\begin{array}{l}\text { Cellule } T \text { attivate, cellule NK, cellule } \\
\text { dendritiche }\end{array}$ & $\begin{array}{l}\text { Espressione MHC dall'endotelio, funzione dei } \\
\text { macrofagi, differenziazione cellule } T \text {, attività NK }\end{array}$ \\
\hline TGF- $\beta$ & Cellule T e B, macrofagi & Fibrosi \\
\hline TNF- $\alpha$ & Macrofagi, cellule T e B, NK & Pro-infiammatoria, citotossicità \\
\hline
\end{tabular}

I macrofagi attivati sono importanti mediatori della sensibilità ritardata che porta a distruzione cellulare. L'attivazione dei macrofagi viene fatta sia dai linfociti CD4 che CD8 attraverso la produzione di IFN- $\gamma$. Il macrofago attivato esercita un effetto citopatico attraverso la produzione di $\mathrm{TNF}-\alpha$, radicali liberi di ossigeno, ed ossido nitrico.

\section{- Risposta immune umorale}

Le immunoglobuline legate alla membrana della cellula B hanno la funzione di recettore dell'antigene e sono conosciute come B-cell receptor (BCR). L'attivazione delle cellule B richiede il legame dell' antigene con il BCR. Per quanto anche alcuni polisaccaridi e proteine polimeriche possano attivare direttamente la cellula B, in generale la risposta anticorpale richiede sia il legame fra antigene e BCR sia l'interazione fra cellula B e cellula T helper specifica per lo stesso antigene. Le cellule $\mathrm{T}$ helper riconoscono frammenti peptidici, li internalizzano e li presentano come complessi peptide-MHC di seconda classe sulla superficie delle cellule B.

Le cellule T helper stimolano le cellule B sia attraverso il legame fra CD40L e CD40 sulle cellule B, sia attraverso la liberazione di citochine.

La secrezione di anticorpi è la principale funzione effettrice delle cellule $B$ nell'immunità adattiva. Le molecole anticorpali hanno due funzioni distinte. Una è di legarsi in modo specifico alle molecole antigeniche che hanno determinato la risposta immune, l'altra è di reclutare altre cellule e molecole per distruggere il patogeno o la cellula esogena legata all'anticorpo.

Le cinque maggiori classi di anticorpi sono: $\operatorname{IgM}, \operatorname{IgD}$, $\operatorname{IgG}, \operatorname{IgA}$, e IgE. Le IgG sono le più abbondanti ed hanno diverse sottoclassi (IgG 1, 2, 3, e 4). La molecola della IgG è formata da due identiche catene leggere (L) e da due identiche catene pesanti $(\mathrm{H})$. Il tutto ha un aspetto ad Y. Ognuna delle 4 catene ha una regione variabile (V) al suo terminale amminoacidico (porzione Fab). Questa regione contribuisce alle strutture atte a legare l'antigene. Il resto della struttura costituisce la parte costante (porzione Fc) e contribuisce a determinare l'isotipo della Ig. La regione V di un determinato anticorpo contiene un segmento ipervariabile che determina la specificità per l'antigene.

Dopo appropriata attivazione, le cellule B secernono IgM e poi si differenziano o in cellule B della memoria o in plasmacellule secernenti anticorpi. Durante questo processo si formano i diversi isotipi anticorpali in risposta alle citochine prodotte dalle cellule $\mathrm{T}$ helper. Sempre le cellule $\mathrm{T}$ helper sono in grado di attivare in modo selettivo mutanti dotati di elevata affinità.

Le funzioni anticorpali differiscono una volta avvenuto il legame con l'antigene. Si può infatti avere fissazione del complemento, opsonizzazione per fagocitosi da parte di cellule contenenti il recettore per Fc, opsonizzazione per lisi cellulare da parte di cellule capaci di esercitare citotossicità cellulare anticorpo-dipendente (cellule NK, macrofagi, neutrofili e eosinofili), e induzione di degranulazione degli eosinofili.

\section{- Linfociti Natural Killer (NK)}

Le cellule NK sono un subset di linfociti periferici che svolgono funzioni simili a quelle dei linfociti CD8. ${ }^{9}$ Le cellule NK periferiche sono mature, non richiedono co-stimolazione e differenziazione, come invece avviene per le cellule $\mathrm{T}$, e rilasciano prontamente granuli citotossici e citochine pro-infiammatorie come TNF- $\alpha$ e IFN- $\gamma$. Poiché hanno una forte potenzialità citolitica e possono 
andare incontro ad autoreattività, la funzione delle cellule NK necessita di essere controllata dall'organismo con estrema precisione. I meccanismi di attivazione sono le citochine, il legame degli anticorpi al recettore Fc, ed il legame dei diversi ligands a recettori attivanti o inibenti.

\section{Conclusioni}

L'organismo è strutturato in modo tale da riconoscere prontamente e rigettare il materiale non-self, sia esso un patogeno esogeno, sia esso materiale biologico proveniente da individui appartenenti alla stessa specie.

Esiste infatti nell'uomo una struttura, chiamata sistema maggiore di istocompatibilità, atta a riconoscere il materiale non-self. Tale struttura, ubicata sulle cellule dell'organismo, è predisposta ad identificare il materiale esogeno con cui viene a contatto. Avvenuto il riconoscimento, si innesca l'immunità adattiva atta, se non controllata, a rifiutare il materiale esogeno. Abbiamo visto che questo fenomeno si svolge attraverso delle tappe precise che iniziano col fenomeno della ischemia-riperfusione. Vengono coinvolti svariati tipi di cellule, principalmente i linfociti $\mathrm{T}$ e $\mathrm{B}$ e le loro sottospecie. Vengono altresì coinvolte molecole dotate di potente attività biologica come le citochine. Il tutto, se non controllato, si conclude col rigetto dell'organo trapiantato.

Tale fenomeno verrà analiticamente descritto nel prossimo numero della rassegna.

\section{Dichiarazione di assenza di conflitto di interessi}

Gli Autori dichiarano di non avere conflitti di interessi.

\section{Finanziamenti}

Gli Autori dichiarano di non aver ricevuto finanziamenti specifici da qualsiasi ente nei settori pubblico, privato o senza fini di lucro.

\section{Referenze}

1. Jones EY. MHC class I and class II structures. Curr Opin Immunol 1997; 9(1):75-79.

2. Zou Y, Stastny P, Süsal C, et al. Antibodies against MICA antigens and kidney-transplant rejection. $N$ Engl $\mathrm{J}$ Med 2007; 357(13):1293-300.

3. Heeger PS and Dinavahi R. Transplant immunology for non-immunologist. Mt Sinai J Med 2012; 79(3):376-387.

4. Huang Y, Rabb H and Womer KL. Ischemia-reperfusion and immediate T cell responses. Cell Immunol 2007; 248(1):4-11.

5. Alegre ML, Lakkis FG and Morelli AE. Antigen Presentation in Transplantation. Trends Immunol 2016; 37(12):831-843.

6. Li XC, Rothstein DM and Sayegh MH. Costimulatory pathways in transplantation: challenges and new developments. Immunol Rev 2009; 229(1):271-293.

7. Willinger T, Freeman T, Hasegawa H, et al. Molecular signatures distinguish human central memory from effector memory CD8 T cell subsets. J Immunol 2005; 175(9): 5895-5903.

8. Buzza MS and Bird PI. Extracellular granzymes: current perspectives. Biol Chem 2006; 387(7):827-837.

9. Young NT. Immunobiology of natural killer lymphocytes in transplantation. Transplantation 2004; 78(1):1-6. 